\title{
Confirmed 6-Month Disability Improvement and Worsening Correlate with Long-term Disability Outcomes in Alemtuzumab-Treated Patients with Multiple Sclerosis: Post Hoc Analysis of the CARE- MS Studies
}

Samuel F. Hunter (D) - Rany A. Aburashed $\cdot$ Raed Alroughani $\cdot$ Andrew Chan $\cdot$ Dominique Dive $\cdot$ Sara Eichau Daniel Kantor · Ho Jin Kim · Jan Lycke · Richard A. L. Macdonell • Carlo Pozzilli • Thomas Scott • Basil Sharrack · Heinz Wiendl · Luke Chung · Nadia Daizadeh · Darren P. Baker · Patrick Vermersch · on behalf of The CARE-MS I, CARE-MS II, CAMMS03409, and TOPAZ Investigators

Received: April 1, 2021 / Accepted: June 3, 2021 / Published online: June 24, 2021

(C) The Author(s) 2021

\section{ABSTRACT}

Introduction: In the 2-year CARE-MS trials (NCT00530348; NCT00548405) in patients with relapsing-remitting multiple sclerosis, alemtuzumab showed superior efficacy versus subcutaneous interferon beta-1a. Efficacy was

Supplementary Information The online version contains supplementary material available at https:// doi.org/10.1007/s40120-021-00262-3.

\section{S. F. Hunter $(\square)$}

Advanced Neurosciences Institute, 101 Forrest

Crossing Blvd., Franklin, TN 37064, USA

e-mail: sfhunter@neurosci.us

R. A. Aburashed

Institute for Neurosciences and Multiple Sclerosis, Owosso, MI, USA

R. Alroughani

Division of Neurology, Department of Medicine,

Amiri Hospital, Sharq, Kuwait

\section{A. Chan}

Department of Neurology, Inselspital Bern, Bern University Hospital, University of Bern, Bern, Switzerland

D. Dive

University Hospital Centre of Liège, Liège, Belgium maintained in two consecutive extensions (NCT00930553; NCT02255656). This post hoc analysis compared disability outcomes over 9 years among alemtuzumab-treated patients according to whether they experienced confirmed disability improvement (CDI) or worsening (CDW) or neither CDI nor CDW.

Methods: CARE-MS patients were randomized to receive two alemtuzumab courses (12 mg/day; 5 days at baseline; 3 days at 12 months), with additional as-needed 3-day

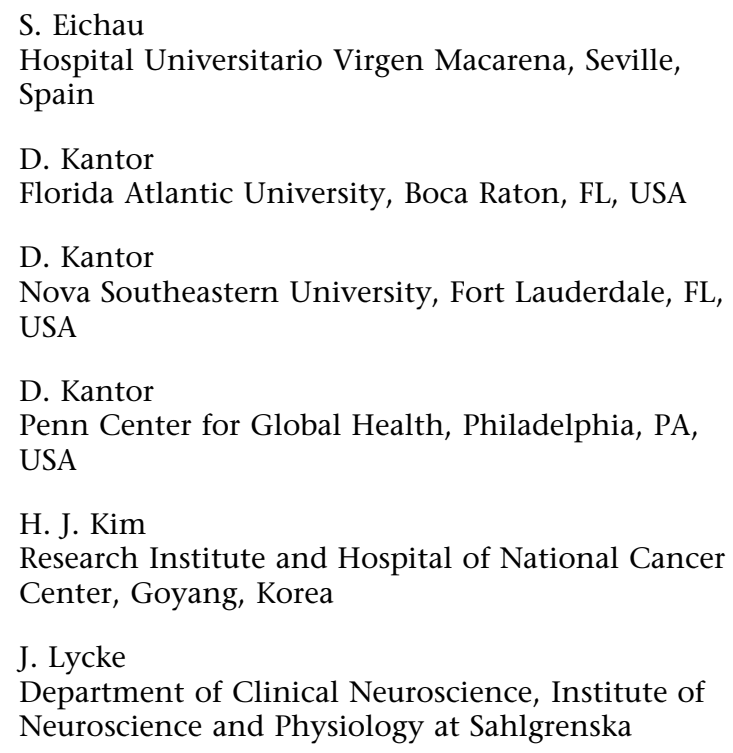
USA

D. Kantor

Penn Center for Global Health, Philadelphia, PA, USA

H. J. Kim

Research Institute and Hospital of National Cancer Center, Goyang, Korea

J. Lycke

Department of Clinical Neuroscience, Institute of Neuroscience and Physiology at Sahlgrenska 
courses in the extensions. CDI or CDW were defined as $\geq 1.0$-point decrease or increase, respectively, in Expanded Disability Status Scale (EDSS) score from core study baseline confirmed over 6 months, assessed in patients with baseline EDSS score $\geq 2.0$. Improved or stable EDSS scores were defined as $\geq 1$-point decrease or $\leq 0.5$-point change (either direction), respectively, from core study baseline. Functional systems (FS) scores were also assessed.

Results: Of 511 eligible patients, 43\% experienced CDI and 34\% experienced CDW at any time through year 9 (patients experiencing both CDI and CDW were counted in each individual group); 29\% experienced neither CDI nor CDW. At year 9, patients with CDI had a -0.58-point mean EDSS score change from baseline; $88 \%$ had stable or improved EDSS scores. Improvements occurred across all FS, primarily in sensory, pyramidal, and cerebellar domains. Patients with CDW had a +1.71 -point mean EDSS score change; $16 \%$ had stable or improved EDSS scores. Patients with neither CDI nor CDW had a -0.10 -point mean EDSS score

Academy, University of Gothenburg, Gothenburg, Sweden

R. A. L. Macdonell

Austin Health and Florey Institute of Neuroscience and Mental Health, Melbourne, VIC, Australia

C. Pozzilli

Department of Human Neuroscience, Sapienza

University, Rome, Italy

T. Scott

Allegheny General Hospital, Drexel University

College of Medicine, Pittsburgh, PA, USA

B. Sharrack

Sheffield NIHR Neuroscience BRC and Sheffield

Teaching Hospitals, Sheffield, UK

H. Wiendl

University of Münster, Münster, Germany change; $98 \%$ had stable or improved EDSS scores.

Conclusion: CDI achievement at any point during the CARE-MS studies was associated with improved disability at year 9, highlighting the potential of alemtuzumab to change the multiple sclerosis course. Conversely, CDW at any point was associated with worsened disability at year 9.

Keywords: Alemtuzumab; Confirmed disability improvement; Confirmed disability worsening; Disease-modifying therapy; Expanded Disability Status Scale; Functional systems; Multiple sclerosis

L. Chung · N. Daizadeh · D. P. Baker

Sanofi, Cambridge, MA, USA

P. Vermersch

Univ. Lille, INSERM U1172 LilNCog, CHU Lille,

FHU Precise, Lille, France 


\section{Key Summary Points}

\section{Why carry out this study?}

Neurologic disability accumulates in patients with relapsing-remitting multiple sclerosis (RRMS) and has a negative impact on their lives, but the correlation between sustained, clinically meaningful disability improvement during RRMS treatment and long-term outcomes is often not fully appreciated.

The CARE-MS studies, which have accrued 9 years of experience with alemtuzumab in treatment-naive and previously treated patients with RRMS, provide data that inform the relationship between confirmed disability improvement (CDI) and confirmed disability worsening (CDW) at any time over the 9 years with long-term disability outcomes.

\section{What was learned from the study?}

Patients with Expanded Disability Status Scale (EDSS) score $\geq 2.0$ at baseline and who had achieved 6-month CDI at any point in the CARE-MS studies showed improved disability outcomes through year 9, and patients with 6-month CDW at any time experienced increased disability through the 9-year follow-up period.

Of the patients who achieved 6-month CDI, a substantial majority had improvements or stabilization in multiple functional systems (FS) of the EDSS, including the pyramidal, cerebellar, and sensory FS, which affect proprioception, mobility, and coordination, and are highly relevant to patient functioning.

The robust and sustained improvements in disability outcomes in patients who achieved 6-month CDI suggest disability can be reduced in patients with higher baseline EDSS scores and indicate a broad, clinically meaningful treatment effect with alemtuzumab.

\section{DIGITAL FEATURES}

This article is published with digital features, including a summary slide, to facilitate understanding of the article. To view digital features for this article go to https://doi.org/10.6084/ m9.figshare. 14710542 .

\section{INTRODUCTION}

Accumulation of neurologic disability negatively impacts the lives of people with multiple sclerosis (MS) $[1,2]$. Preventing disability progression and, more importantly, improving preexisting disability over the long term remain important treatment goals. It is well established that initiating disease-modifying therapy (DMT) earlier rather than later in the disease course delays disability progression [3-5]; however, it is not always appreciated how disability improvement or worsening over a defined period (e.g., 6 months) contributes to long-term outcomes.

Alemtuzumab (Lemtrada ${ }^{\circledR}$, Sanofi Genzyme, Cambridge, MA, USA) showed superior efficacy on relapse and magnetic resonance imaging (MRI) outcomes versus subcutaneous interferon beta-1a (SC IFNB-1a; Rebif ${ }^{\circledR}$, EMD Serono, Inc., Rockland, MA) over 2 years in the Comparison of Alemtuzumab and Rebif ${ }^{\circledR}$ Efficacy in Multiple Sclerosis (CARE-MS) studies in patients with relapsing-remitting MS (RRMS) [6, 7]. In addition, a significantly greater percentage of alemtuzumab-treated patients in CARE-MS II who had received prior DMT were free of 6-month confirmed disability worsening (CDW) and achieved 6-month confirmed disability improvement (CDI) versus SC IFNB-1a [7, 8]. Alemtuzumab maintained its clinical efficacy over an additional 7 years in two consecutive extension studies, the CARE-MS extension $[9,10]$ and the ongoing TOPAZ extension study [11-13]. The underlying mechanism for these improvements in disability is not fully understood, but could be due to structural and functional repair within the central nervous system $[8,14]$. 
CDI has been used as an outcome measure in DMT trials to assess reversal of patients' preexisting disability [7-10, 15-19]. By requiring a confirmation of Expanded Disability Status Scale (EDSS) score change over 6 months, CDI reflects a sustained and clinically meaningful improvement in patient functioning, rather than simple recovery from relapse.

We report an analysis of the pooled CAREMS population that evaluated disability status in alemtuzumab-treated patients who had 6-month CDI, 6-month CDW, or neither CDI nor CDW over 9 years, and characterized improvement in individual functional systems (FS) scores of the EDSS over 6 years.

\section{METHODS}

This pooled analysis included patients from CARE-MS I and II randomized to receive two courses of alemtuzumab $12 \mathrm{mg} /$ day (5 days at baseline; 3 days at 12 months), with additional as-needed 3-day courses in the extensions. Patients were stratified into three subgroups: with CDI, with CDW, and with neither CDI nor CDW. Any patient who experienced both CDI and CDW during the 9-year period was counted in both the subgroup of patients with CDI and the subgroup of patients with CDW. EDSS and FS scores, as well as annualized relapse rate (ARR) and quality-of-life (QoL) outcomes, were assessed. Safety events were recorded through year 9 .

\section{Study Design}

This post hoc analysis included only those patients receiving alemtuzumab $12 \mathrm{mg}$ in the 2-year core CARE-MS I and II trials. Patients who completed the 2-year core trials could enroll in the two consecutive extension studies: the 4-year CARE-MS extension study (CAMMS03409) and the ongoing TOPAZ extension study. Data are currently available for a total of 9 years for patients treated with alemtuzumab. Results from CARE-MS I and II were pooled for analysis.

Details on the rater-blinded, active-controlled CARE-MS trials and subsequent extension studies have been published previously $[6,7,9-13]$. After $\geq 30$ days of clinical stability, patients were randomized to receive either two courses of alemtuzumab $12 \mathrm{mg}$ /day (given as intravenous [IV] infusions on 5 consecutive days at baseline and on 3 consecutive days 12 months later) or SC IFNB-1a $44 \mu$ g three times per week after dose titration. Patients in both groups received IV methylprednisolone $1 \mathrm{~g} /$ day on three consecutive days at baseline and at month $12[6,7]$. Alemtuzumab-treated patients who entered the 4-year CARE-MS extension study could receive additional courses of alemtuzumab ( $12 \mathrm{mg} /$ day on 3 consecutive days $\geq 12$ months after the most recent dose) as needed for clinical or magnetic resonance imaging (MRI) disease activity or receive other licensed DMTs at the investigator's discretion $[9,10]$. Follow-up of patients beyond 6 years is continuing in the TOPAZ extension study in which patients can receive additional alemtuzumab courses (12 mg/day on 3 consecutive days $\geq 12$ months after the most recent course) or other DMTs at any time point, both at the investigator's discretion (no criteria) [11-13].

\section{Compliance with Ethics Guidelines}

The CARE-MS I and II studies, the CARE-MS extension, and the TOPAZ extension were registered with ClinicalTrials.gov (NCT00530348, NCT00548405, NCT00930553, and NCT02255656, respectively). The studies were conducted in accordance with the ethical principles outlined in the Declaration of Helsinki. The study protocol, informed consent forms, and other study-related documents were reviewed and approved by the local independent ethics committees and institutional review boards. Written informed consent to participate in the studies was obtained from all patients.

\section{6-Month CDI and CDW Assessments Over 9 Years}

This study evaluated pooled CARE-MS I and II alemtuzumab-treated patients over 9 years, and the analysis population for 6-month CDI, 
6-month CDW, or neither 6-month CDI nor 6-month CDW consisted of patients with baseline EDSS scores $\geq 2.0$. Although the entire study population was eligible for CDW analysis, a smaller cohort of patients with EDSS score $\geq 2.0$ was analyzed for CDW to ensure consistency of population with those eligible for CDI. CDI and CDW were defined as $\mathrm{a} \geq 1$.0-point decrease and increase, respectively, in EDSS score from core study baseline confirmed over 6 months. This stringent definition ensured that only those patients with an improvement or worsening of preexisting disability at core study baseline would be classified as having CDI or CDW, respectively. Mean EDSS scores were assessed quarterly from core study baseline through year 6 (end of the CARE-MS extension) and every 6 months over years 7-9 (TOPAZ). Improved and stable EDSS scores were defined as $\mathrm{a} \geq 1$.0-point decrease and $\mathrm{a} \leq 0.5$-point change in either direction, respectively, from core study baseline at a single time point (i.e., did not require confirmation over time) and were assessed annually over 9 years; results at year 9 are reported here. To assess the impact of baseline EDSS score on CDI and CDW, all patients with 6-month CDI, CDW, or neither CDI nor CDW were stratified by core study baseline EDSS scores of 2.0-2.5, 3.0-3.5, 4.0-4.5, 5.0-5.5, and 6.0-6.5.

\section{FS Assessments Over 6 Years}

Individual FS scores were assessed at core study baseline and quarterly through year 6 by raters blinded to treatment. FS scores were collected until the end of the CARE-MS extension study, but not documented as part of the ongoing TOPAZ study. The FS scores were rated on an ordinal scale: cerebellar, cerebral, and brainstem (range 0-5); pyramidal, sensory, bowel/bladder, and visual (range 0-6) [20]. Improvement in FS scores was defined as $\mathrm{a} \geq 1$.0-point decrease from core study baseline; worsening was defined as $\mathrm{a} \geq 1.0$-point increase. Stability in FS scores was defined as a 0-point change from core study baseline. Improvement, worsening, or stability in FS scores since core study baseline was assessed annually over 6 years; this manuscript reports results at year 6 .

\section{QoL Assessments Over 9 Years}

QoL outcomes for alemtuzumab-treated patients, followed from CARE-MS core study baseline through completion of year 9 , were collected and stratified by 6-month CDI, CDW, or neither CDI nor CDW. Four outcome measures were assessed. The Short Form-36 (SF-36) mental component summary (MCS) and physical component summary (PCS) are validated generic self-assessment questionnaires that capture patients' perceptions of their mental and physical health, respectively, and how they affect their QoL [21]. Norm-based scores are utilized for both the MCS and PCS, calibrating each scale to have a mean of 50 and SD of 10 in the general US population [22]. The SF-36 was administered at baseline, every 12 months during the core study, every 6 months during year 1 of the extension period, and every 12 months thereafter [23]. The EuroQol-5 Dimension visual analog scale (EQ-5D VAS) is a validated, standardized, self-assessed generic measure of current health status that is scored on a visual analog scale ranging from 0 (worst imaginable health state) to 100 (best imaginable health state) [24]. The EQ-5D VAS was administered at baseline, every 6 months during the core study and year 1 of the extension period, and every 12 months thereafter [23]. The Functional Assessment of Multiple Sclerosis (FAMS) is a validated, MS-specific, patient-reported healthrelated QoL questionnaire with 44 scored items comprising six scales (mobility, symptoms, emotional well-being, general contentment, thinking/fatigue, family/social well-being); total scores range from 0 to 176 , with higher scores indicating better functioning [25]. The FAMS was administered at baseline, every 6 months during the core study and year 1 of the extension period, and every 12 months thereafter [23]. Worsening and improvement in QoL outcomes were defined as $\geq 5$-point decrease and $\geq 5$-point increase, respectively, from core study baseline; this manuscript reports results at year 9. 


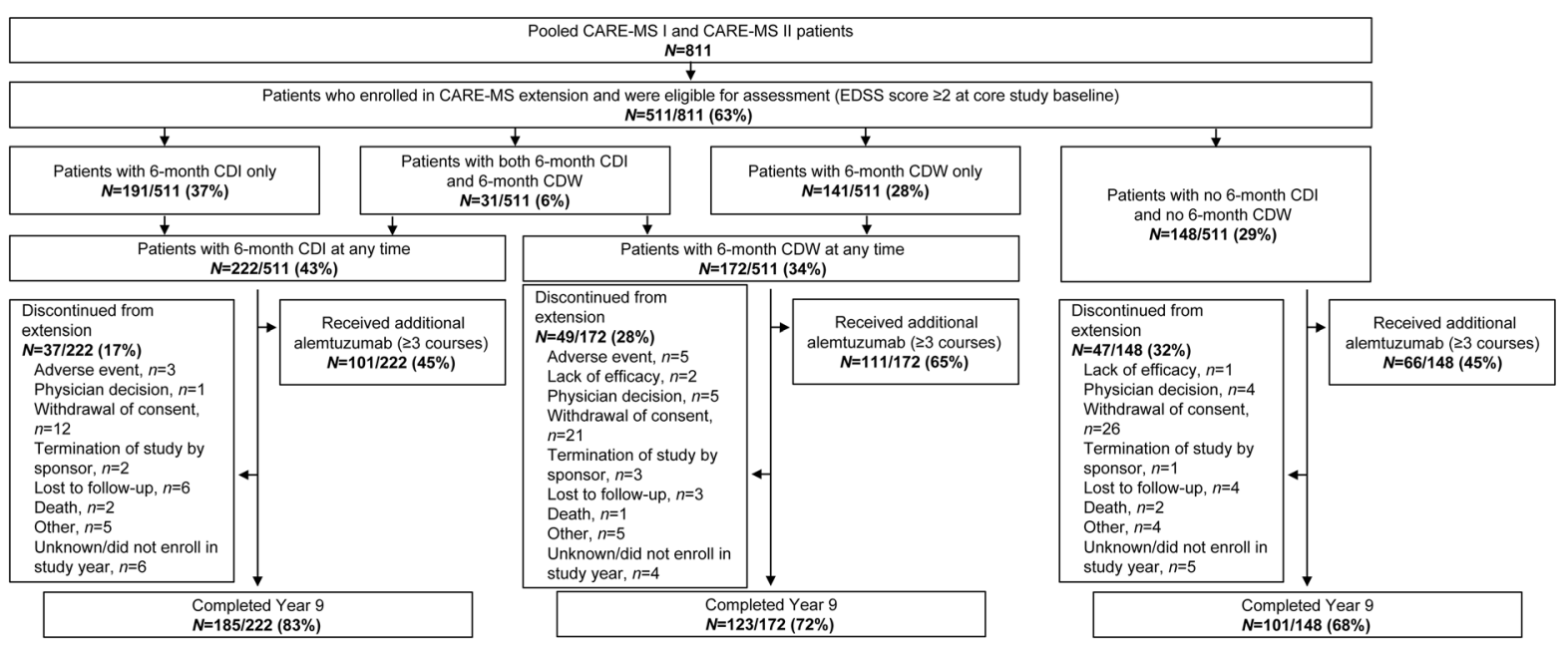

Fig. 1 Disposition of pooled CARE-MS I and II patients stratified by 6-month CDI, 6-month CDW, or neither CDI nor CDW over 9 years. CDI and CDW were defined as $\mathrm{a} \geq 1.0$-point decrease and $\mathrm{a} \geq 1.0$-point increase, respectively, in EDSS score from core study baseline confirmed over 6 months, and were assessed only in

\section{Statistical Analysis}

This analysis was based on all available data (without imputation) from all patients who received alemtuzumab $12 \mathrm{mg}$ in the pooled CARE-MS studies for up to 9 years of follow-up. Patients who received SC IFNB-1a in the core CARE-MS studies and switched to alemtuzumab in the extension study were not included since they were unlikely to provide additional insight into the effects of CDI and CDW on long-term outcomes, as sufficient alemtuzumab-only patients were available. No formal statistical testing was conducted to compare the various subgroups.

\section{RESULTS}

\section{Patients and Disability Events Over 9 Years}

In the pooled analysis of 811 alemtuzumabtreated patients from the CARE-MS I and II studies, 511 patients (63\%) had baseline EDSS scores $\geq 2$ and were included in this analysis (Fig. 1). The baseline demographic and disease patients with EDSS score $\geq 2.0$ at core study baseline. Patients who experienced both CDI and CDW events during the 9-year period were counted in both groups. $C D I$ confirmed disability improvement, $C D W$ confirmed disability worsening, EDSS Expanded Disability Status Scale

characteristics observed across subgroups of patients who experienced CDI, CDW, or neither CDI nor CDW at any time during the 9-year period are shown in Table 1. Most patients were in their fourth decade at study entry, and the population was predominantly female. Differences in baseline characteristics between the cohorts of patients with CDI and CDW included duration of disease and number of gadolinium (Gd)-enhancing lesions at baseline. The mean (SD) time since first symptoms for patients with CDW was 4.0 (2.8) years, compared with 3.6 (2.5) years for patients with CDI, and 3.4 (2.4) years for patients with neither CDI nor CDW. Patients with CDW also had fewer Gd-enhancing lesions at baseline (mean 2.0, SD 4.3) compared with those with CDI (mean 2.6, SD 6.9) or neither CDI nor CDW (mean 3.2, SD 7.6) (Table 1).

Over 9 years, $222 / 511$ patients (43\%) achieved 6-month CDI with a total of 365 events (mean duration 27.2 months), 172/511 patients (34\%) experienced 6-month CDW with a total of 217 events (mean duration 31.3 months), and $148 / 511$ patients (29\%) had neither CDI nor CDW (Fig. 1 and 
Table 1 Baseline characteristics of patients with 6-month CDI, 6-month CDW, or neither CDI nor CDW over 9 years

\begin{tabular}{llll}
\hline Baseline characteristic & $\begin{array}{l}\text { Patients with 6-month } \\
\text { CDI }(\boldsymbol{n}=\mathbf{2 2 2})\end{array}$ & $\begin{array}{l}\text { Patients with 6-month } \\
\text { CDW }(\boldsymbol{n}=\mathbf{1 7 2})\end{array}$ & $\begin{array}{l}\text { Patients with neither CDI nor } \\
\text { CDW }(\boldsymbol{n}=\mathbf{1 4 8})\end{array}$ \\
\hline Age, years & $34.5(8.1)$ & $36.5(8.1)$ & $33.3(7.9)$ \\
Female, $n$ (\%) & $150(68)$ & $114(66)$ & $93(63)$ \\
Time since first symptoms, years & $3.6(2.5)$ & $4.0(2.8)$ & $3.4(2.4)$ \\
Relapses in previous year & $1.7(0.9)$ & $1.7(0.8)$ & $1.7(0.8)$ \\
$\begin{array}{l}\text { Relapses in previous 2 years } \\
\text { EDSS score }\end{array}$ & $2.6(1.0)$ & $2.6(1.0)$ & $2.7(1.2)$ \\
$\begin{array}{l}\text { Number of Gd-enhancing } \\
\text { lesions }\end{array}$ & $3.0(0.8)$ & $3.1(1.0)$ & $2.7(0.7)$ \\
$\begin{array}{l}\text { Patients with baseline Gd- } \\
\text { enhancing lesions, } n(\%)\end{array}$ & $100(46)^{\mathrm{a}}$ & $2.0(4.3)^{\mathrm{b}}$ & $3.2(7.6)^{\mathrm{c}}$ \\
$\begin{array}{l}\text { T2 hyperintense lesion volume, } \\
\mathrm{cm}^{3}\end{array}$ & $9.8(13.0)^{\mathrm{d}}$ & $71(42)^{\mathrm{b}}$ & $59(40)^{\mathrm{c}}$ \\
\begin{tabular}{l} 
Brain parenchymal fraction \\
\hline
\end{tabular} & $0.82(0.02)^{\mathrm{f}}$ & $10.9(13.5)^{\mathrm{e}}$ & $9.9(10.6)^{\mathrm{c}}$ \\
\hline
\end{tabular}

Values are mean (SD) except where noted. CDI and CDW were defined as a $\geq 1.0$-point decrease and a $\geq 1.0$-point increase, respectively, in EDSS score from core study baseline confirmed over 6 months, and were assessed only in patients with EDSS score $\geq 2.0$ at core study baseline

$C D I$ confirmed disability improvement, $C D W$ confirmed disability worsening, EDSS Expanded Disability Status Scale, $G d$ gadolinium

a $n=218$

b $n=170$

c $n=147$

d $n=220$

e $n=171$

$f_{n}=217$

g $n=169$

${ }^{\text {h }} n=146$

Supplementary Material). The proportions of patients in each category exceeded 100\% because $31 / 511$ patients $(6 \%)$ had both CDI and CDW events during the 9-year period and were counted in both groups. For the 222 patients who achieved 6-month CDI, 65\% had an event during the first 2 years (Supplementary Material). Of the 31 patients who had both CDI and CDW events, 26 had CDW after CDI (i.e., $11.7 \%$ [26/222] of patients who achieved CDI subsequently had a CDW), and 5 achieved CDI after CDW (i.e., 2.9\% [5/172] of patients who had a CDW subsequently had a CDI). The proportion of patients who received additional alemtuzumab courses was lower for those with CDI (101 patients [45.5\%]; comprising 57 [25.7\%], 29 [13.1\%], 11 [5.0\%], 3 [1.4\%], and 1 [0.5\%] who received $1,2,3,4$, or 5 additional courses, respectively) than for those with CDW (111 patients [64.5\%]; comprising 55 [32.0\%], 33 [19.2\%], 15 [8.7\%], 7 [4.1\%], and 1 [0.6\%] who received $1,2,3,4$, or 5 additional courses, respectively; Fig. 1). A higher proportion of patients with CDI than with CDW remained on study through year 9 (83\% vs. $72 \%$; Fig. 1$)$. Among both the subgroup with CDI and the 

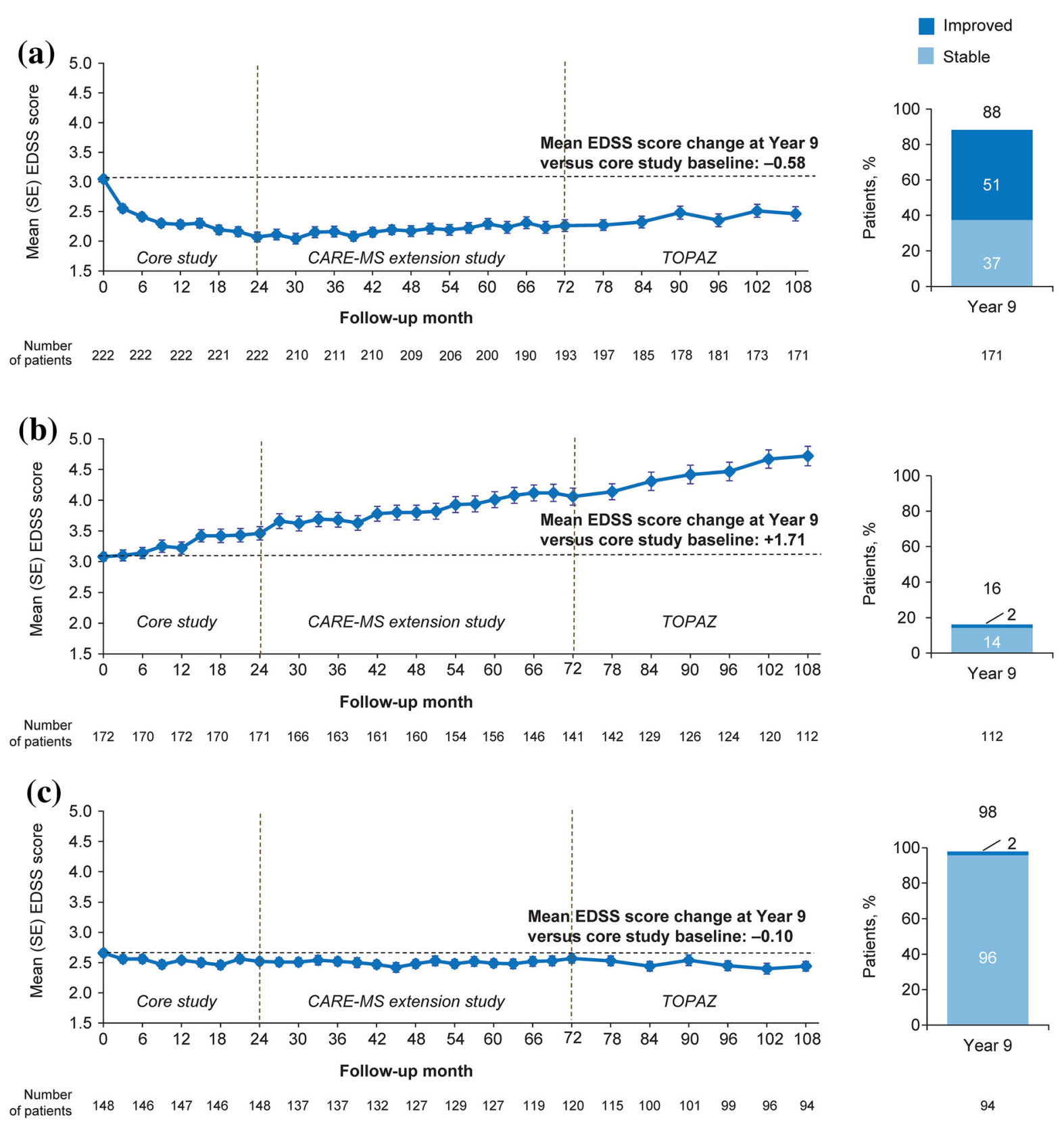

Fig. 2 EDSS scores over 9 years in patients stratified by a 6-month CDI, b 6-month CDW, or c neither CDI nor CDW. Line charts represent EDSS scores through 9 years and bar charts represent improvement or stability in EDSS scores at year 9 relative to core study baseline, both in pooled CARE-MS I and II patients who had 6-month CDI, 6-month CDW, or neither CDI nor CDW at any time during the 9-year study. CDI and CDW were defined as $\mathrm{a} \geq 1.0$-point decrease and $\mathrm{a} \geq 1.0$-point increase, respectively, in EDSS score from core study baseline confirmed over 6 months, and were assessed only in patients with EDSS score $\geq 2.0$ at core study baseline. Improved and stable EDSS scores were defined as $\geq 1$ point decrease and $\leq 0.5$-point change in either direction, respectively, from core study baseline at a single time point (year 9), and did not require confirmation over time. CDI confirmed disability improvement, $C D W$ confirmed disability worsening, EDSS Expanded Disability Status Scale 
Improved

Stable

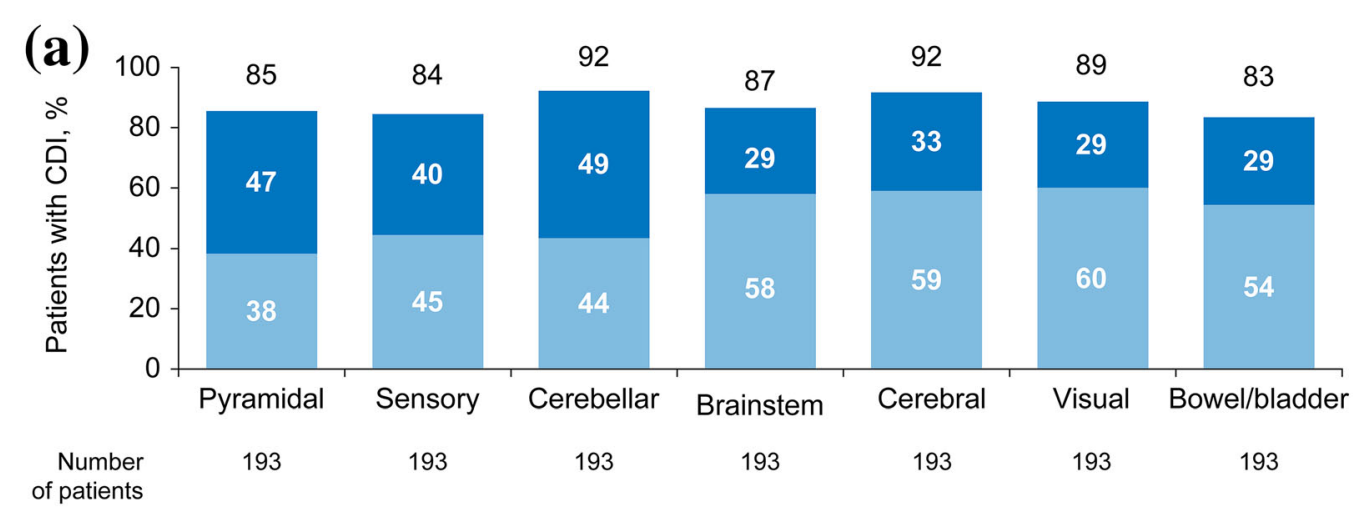

(b)
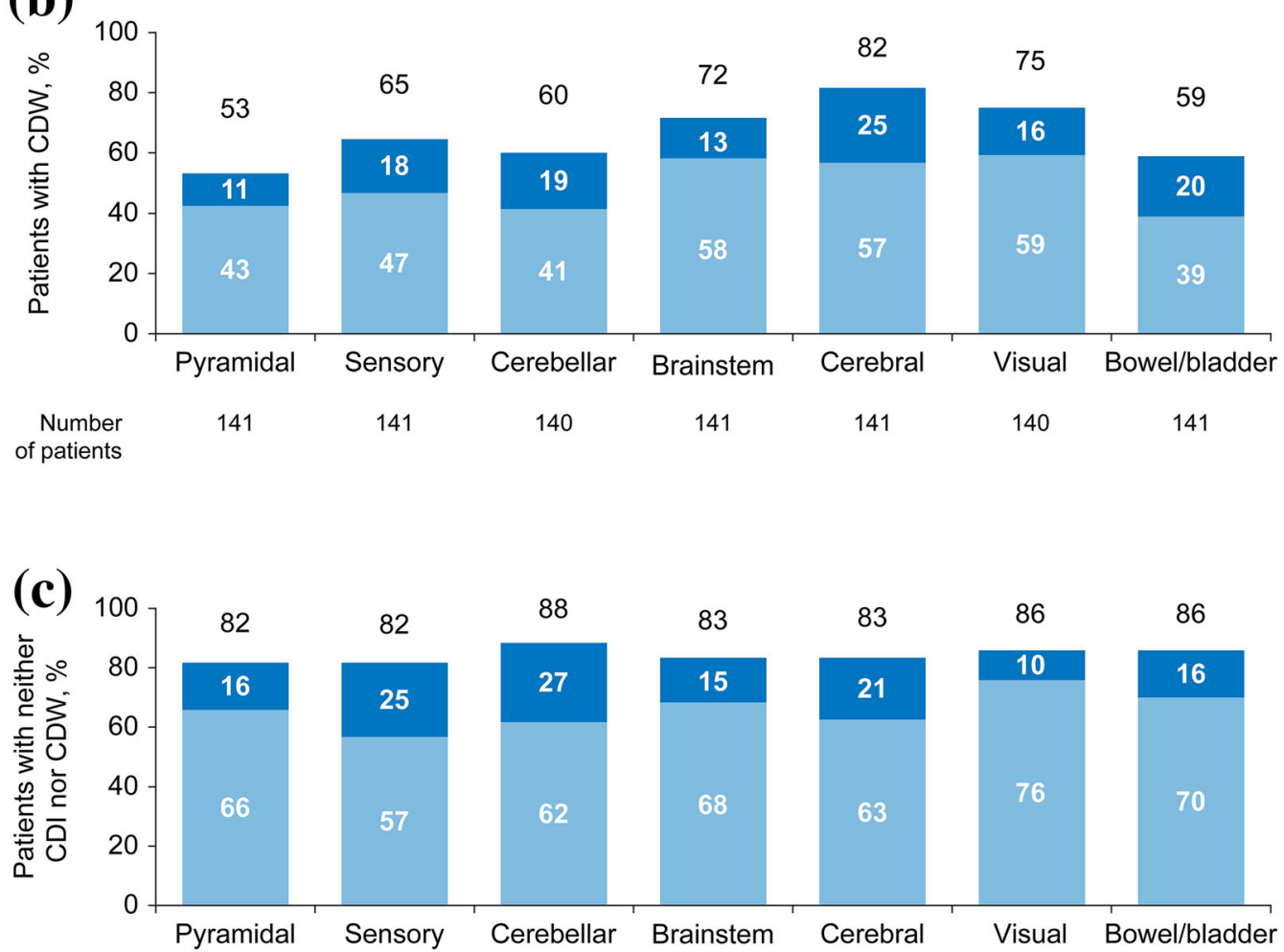

$\begin{array}{llllllll}\text { Number } & 120 & 120 & 120 & 120 & 120 & 120 & 120\end{array}$ 
4Fig. 3 FS scores over 6 years in patients stratified by a 6month CDI, b 6-month CDW, or c neither CDI nor CDW. Includes pooled CARE-MS I and II patients who had 6-month CDI, 6-month CDW, or neither CDI nor CDW at any time during the 9-year study. CDI and CDW were defined as $a \geq 1.0$-point decrease and $a \geq 1.0$-point increase, respectively, in EDSS score from core study baseline confirmed over 6 months, and were assessed only in patients with EDSS score $\geq 2.0$ at core study baseline. FS scores were collected through the end of the CARE-MS extension (year 6), and were not recorded in the TOPAZ extension (years 7-9). Improved and stable FS scores were defined as $\geq 1$-point decrease and $\leq 1$-point change in either direction, respectively, from core study baseline at a single time point (year 6), and did not require confirmation over time. Sum of percentages with improved and stable FS scores may differ due to rounding. $C D I$ confirmed disability improvement, $C D W$ confirmed disability worsening, EDSS Expanded Disability Status Scale, FS functional systems

subgroup with CDW, patients were most likely to have had a baseline EDSS score of either $2.0-2.5$ (36\% of the CDI group; $42 \%$ of the CDW group) or $3.0-3.5$ (44\% of the CDI group; $36 \%$ of the CDW group) (Supplementary Material).

\section{EDSS Scores in Patients Stratified by CDI or CDW Status}

Patients who achieved CDI at any time on study had a mean EDSS score change of -0.58 at year 9 versus core study baseline (Fig. 2a). Overall, 88\% of patients achieving CDI had EDSS scores that remained stable or improved at year 9 relative to baseline (Fig. 2a). Disability improvements were also assessed in patients with a pre-CDI EDSS score above or below 4.0, a threshold associated with moderate to severe MS. Before the onset of CDI, $85 \%$ of patients had EDSS scores $<4.0$, which increased to $97 \% 6$ months after achieving CDI, indicating an improvement in disability at this EDSS milestone. Acknowledging the lower retention rate for patients with CDW than for patients with CDI, the CDW group experienced worsened disability at year 9 compared with the CDI group, with a +1.71 mean change in EDSS score from core study baseline, and $16 \%$ had stable or improved EDSS scores at year 9 relative to baseline (Fig. 2b). EDSS scores remained stable at year 9 relative to baseline in $96 \%$ of patients who had neither CDI nor CDW, and an additional $2 \%$ of patients had improved EDSS scores. This subgroup had a -0.10 mean change in EDSS score from core study baseline (Fig. 2c).

\section{FS Scores in Patients Stratified by CDI or CDW Status}

Among patients with CDI, confirmed improvements occurred across all FS reported through the end of the CARE-MS extension study (year 6), with the most frequent improvements occurring in the cardinal FS domains of pyramidal (47\%), sensory (40\%), and cerebellar (49\%). Overall, $29-33 \%$ of patients with CDI showed improvements in the brainstem, cerebral, visual, and bowel/bladder FS (Fig. 3a). Rates of improvement in $1,2,3$, and $\geq 4$ FS were $23 \%, 23 \%, 24 \%$, and $24 \%$, respectively (Supplementary Material). Compared with patients with CDI, lower percentages of patients with CDW had improved or stable scores across all FS, with the lowest rate of improvement observed in the pyramidal FS (11\%) (Fig. 3b). A total of $34 \%$ of patients with CDW showed no improvements in any FS, compared with $6 \%$ of patients with CDI, and $3-11 \%$ of patients with CDW showed improvements in $\geq 3$ FS, compared with $24 \%$ of those with CDI (Supplementary Material). Among patients with neither CDI nor CDW, 57-76\% had stable FS scores at year 6 relative to core study baseline (Fig. 3c). Improvements were observed in multiple FS in this subgroup of patients, with $37 \%, 20 \%, 14 \%$, and $3 \%$ showing improvements in 1, 2, 3, and $\geq 4 \quad F S$, respectively (Supplementary Material).

\section{ARR in Patients Stratified by CDI or CDW Status}

Cumulatively over years 0-9, ARR (95\% CI) was $0.159(0.133,0.190), 0.308(0.270,0.351)$, and $0.167(0.135,0.207)$ in patients with CDI, patients with CDW, and patients with neither 
CDI nor CDW, respectively (Supplementary Material). During each year from year 1 to year 9, the ARR was consistently higher in patients with CDW compared with the other two patient subgroups (Supplementary Material).

\section{QoL Scores in Patients Stratified by CDI or CDW Status}

Over 9 years, $34 \%$ and $37 \%$ of patients with CDI had improvements on the SF-36 MCS and PCS, respectively. Rates of improvements were $49 \%$ on the EQ-5D VAS and $56 \%$ on the FAMS (Supplementary Material). Lower proportions of patients with CDW had improved QoL scores across all assessments (SF-36 MCS, 31\%; SF-36 PCS, 14\%; EQ-5D VAS, 33\%; FAMS, 28\%) over 9 years (Supplementary Material). Similarly, lower proportions of patients with neither CDI nor CDW showed improvements across QoL assessments compared with those with CDI, with the exception of SF-36 MCS (Supplementary Material).

\section{Safety}

Rates of adverse events (AEs), including infections and autoimmune AEs, were similar for patients with 6-month CDI, 6-month CDW, or neither CDI nor CDW (Supplementary Material). Overall, thyroid AEs were more common in the CDI group (55\%) than in the CDW (49\%) or neither CDI nor CDW group (41\%), but rates of serious thyroid AEs were similar for the three groups. Compared with those who achieved CDI, patients with CDW had an apparent increased incidence of serious AEs (54\% vs. $45 \%$ ) and deaths ( $4 \%$ vs. $2 \%$ ). Malignancy rates remained low across all subgroups (1-2\%).

\section{DISCUSSION}

For treatment-refractory patients with MS, decisions on subsequent therapy are driven in the first instance by the goals of stabilizing disability levels and preventing further worsening of disability. A further target is improvement of patients' disability status, and the findings from our study support the robustness of the relatively novel outcome parameter CDI. However, substantial variability in disease course within and among patients complicates disability assessments. Moreover, biases in patient retention complicate interpretation of long-term disability outcomes beyond the typical 1-3 years of follow-up in randomized trial assessments [26]. Previous studies that have conceptualized the natural history of MS as a two-phase disease have suggested that patients with advanced disease (i.e., EDSS score $\geq 4.0$ ) do not benefit from therapeutic intervention because their disease is mainly neurodegenerative and independent of inflammation [27, 28]. The contemporary view of MS disease trajectory, however, suggests that disability accumulation is driven by relapses (i.e., inflammatory events) and brain atrophy at any time in the disease course, leading to highly variable treatment outcomes in patients with advanced disease [29-31]. Sustained improvements in preexisting disability with alemtuzumab, even among patients with higher baseline EDSS scores, are consistent with this view and emphasize the importance of continuing highly effective treatment in advanced MS. The nonlinearity of the EDSS rating makes it insensitive to measurements other than gait disturbances in patients with scores $\geq 4.0$ [32]. Hence, in these patients, FS score improvements may have a greater clinical impact than numerical improvements in EDSS scores. Interestingly, the rates of CDI in the post hoc analysis reported here were $44 \%$ in patients with baseline EDSS scores of 3.0-3.5 compared with 36\% for patients with baseline EDSS scores of 2.0-2.5, and $69 \%$ of the former had improvement or stability in $\geq 2$ FS (data not shown). Although the underlying mechanism of improving preexisting disability based on EDSS is unclear, studies have shown a proportional association between the magnitude of ongoing inflammatory activity and axonal injury (i.e., neurodegeneration) in all disease stages of MS [33].

Most importantly, $88 \%$ of alemtuzumab patients who achieved 6-month CDI continued to show improved $(51 \%)$ or stable $(37 \%)$ EDSS scores at year 9 compared with study baseline. This is in contrast to patients with CDW, of 
whom $16 \%$ had improved $(2 \%)$ or stable $(14 \%)$ EDSS scores at year 9. The majority of patients (96\%) with neither CDI nor CDW had stable EDSS scores; 2\% had improved EDSS scores at year 9 .

The burden of Gd-enhancing lesions at baseline was greater for patients with CDI or with neither CDI nor CDW compared with patients with CDW. This could suggest that patients with lower inflammatory activity (as measured by fewer Gd-enhancing lesions) have a more advanced disease course and may have poorer outcomes than patients presenting with earlier stages of RRMS, and supports the rationale for the treatment of early MS with alemtuzumab [34]. Alternatively, these findings could also suggest that patients with advanced focal inflammatory disease may be better responders to a highly effective treatment, such as alemtuzumab. To this end, previous studies have suggested that long-term disability improvements with alemtuzumab may not be solely due to its anti-inflammatory effect, but may be also attributable to a neuroprotective effect via neurotrophin secretion from immune cells regenerating after alemtuzumab treatment [35].

The FS of the EDSS play a key role in defining disease progression in patients with MS [36]. In a typical cohort of patients with MS, the pyramidal, cerebellar, and sensory FS tended to be most affected at baseline, yet were also the most likely FS to improve in response to treatment $[36,37]$. Of the alemtuzumab-treated patients who achieved 6-month CDI, 72\% showed improvements in $\geq 2 \mathrm{FS}$, including in the pyramidal (47\%), cerebellar (49\%), and sensory (40\%) FS. These findings are consistent with previous observations from the phase 2 CAMMS223 trial, showing greatest improvements with alemtuzumab versus SC IFNB-1a for pyramidal, cerebellar, and sensory FS [38]. These FS affect proprioception, mobility, and coordination, and are highly relevant to patient functioning. Given the negative impact of functional and mobility impairments on the QoL of patients with MS [2], these robust and sustained improvements in each FS suggest that dysfunction can be repaired in patients with higher baseline EDSS scores, and indicate a broad and clinically meaningful treatment effect with alemtuzumab.

In this analysis, QoL endpoints mirrored long-term disability outcomes, with higher percentages of patients with 6-month CDI versus 6-month CDW achieving improvements in SF-36 MCS, SF-36 PCS, EQ-5D VAS, and FAMS scores. Over 9 years of follow-up, the safety profile of alemtuzumab was generally similar in patients with CDI, CDW, or neither CDI nor CDW. Differences were noted, however, in rates of serious AEs for patients with CDW (54\%) compared with patients with CDI (45\%) and neither CDI nor CDW (38\%), which is perhaps expected given that, by definition, patients with 6-month CDW have poorer outcomes.

The search for prognostic factors that contribute to long-term outcomes in patients with RRMS remains an active area of ongoing research. The concept of disability status changes during treatment is increasingly recognized as a clinically relevant endpoint in recent studies [39-41]. One key strength of this analysis is the large sample size from two randomized, active-controlled, rater-blinded, phase 3 studies with a well-defined population of both treatment-naive and previously treated patients with RRMS. This analysis also presents data for both patients with improved disability and worsened disability. The $83 \%$ retention rate of patients with CDI, $72 \%$ of CDW, and $68 \%$ of neither CDI nor CDW through year 9 in the TOPAZ extension study provides further support for the robustness of this analysis. Although improvements in preexisting disability have been reported with other DMTs, they were in placebo-controlled trials using a less stringent definition of CDI (3-month CDI, 0.5point EDSS improvement) and a shorter duration of follow-up [15-18]. One recently reported study followed 1882 patients with RRMS for a median of 1.6 years and found that CDI rates were $11 \%$ for ofatumumab and $8 \%$ for teriflunomide (hazard ratio 1.35, $P=0.09$ ) [39].

The main limitation of this study is its post hoc nature, the inter-rater variability in EDSS and FS score assessments, the widely acknowledged weighting of ambulation in EDSS scoring and insensitivity to cognitive dysfunction, and the relatively small sample sizes of the 
subgroups stratified by baseline EDSS score. Nevertheless, the EDSS is a rigorous and valuable tool to capture neurologic disability in patients, recognized as an important component in evaluating comparative DMT efficacy in MS trials. A further limitation is that the factors that define the patient subgroups in this study (presence of CDI or CDW), as well as some of the outcomes assessed, share EDSS score as a common source, which precludes the possibility of statistical comparisons being performed between the patients with CDI or CDW.

\section{CONCLUSIONS}

Achieving continuous and sustained disability improvements across the FS of the EDSS for many years after initiation of DMT is an important treatment goal in MS, as it directly affects daily functioning. To this end, the EDSS reflects only the most severely impaired FS scores, and improvements in multiple less impaired domains may go unnoticed on the EDSS in disabled patients. Although long-term biases in follow-up tend to favor treatment successes, the long-term retention and improvements present in even some of the patients who had experienced instances of CDW in our study indicate that these findings reflect meaningful disability improvements over time. The improvement in multiple aspects of disability in patients with RRMS over 9 years highlights the potential for alemtuzumab to change the MS disease course.

\section{ACKNOWLEDGEMENTS}

The authors and Sanofi thank the patients for their participation in the trials, as well as the CARE-MS I and CARE-MS II Steering Committees and the CAMMS03409 and TOPAZ Investigators (the names of the investigators are found within the Supplementary Material).

Funding. The study was supported by Sanofi and Bayer HealthCare Pharmaceuticals. The sponsor was involved in the design and conduct of the study; collection, management, analysis, and interpretation of the data; and preparation, review, or approval of the manuscript. The journal's Rapid Service Fee was provided by Sanofi.

Editorial Assistance. Editorial support was provided by Panos Xenopoulos, PhD, of Elevate Scientific Solutions, and was funded by Sanofi.

Authorship. All authors meet the International Committee of Medical Journal Editors (ICMJE) criteria for authorship for this article. All authors had full access to the study data and take responsibility for the integrity of the data and the accuracy of the data analysis, and have given their approval for this version to be published.

Authors' Contributions. Author contributions for this study are as follows: conception and design (DPB, SFH, JL, CP, BS, TFS), statistical analysis (ND), interpretation of data (all authors), and drafting the manuscript (all authors). All authors have read and given their approval for this version to be published, and agree to be accountable for all aspects of the work.

Prior Presentation. Some of the data in the manuscript were presented in poster \#DXT65 titled: "Longitudinal Disability Follow-up in Patients With 6-Month Confirmed Disability Improvement or Worsening in the CARE-MS and Extension Studies" at the 2020 Virtual Annual Meeting of the Consortium of Multiple Sclerosis Centers (CMSC), August 3rd 2020.

Disclosures. Samuel F. Hunter reports consulting agreements, speaker honoraria, and grant/research financial support from AbbVie, Avanir, Bayer HealthCare, Biogen, Janssen, Mallinckrodt, Novartis, Osmotica, Roche, Sanofi, and Teva. Rany A. Aburashed reports compensation for serving as a consultant, speaker, and advisory board participant from Bayer, Biogen, Genentech, Sanofi, and Teva; and research grants from Novartis. Raed Alroughani reports receiving honoraria as a speaker and scientific advisory board 
participant, and research grants from Bayer, Biogen, Biologix, Genpharm, GlaxoSmithKline, Lundbeck, Merck Serono, Novartis, Roche, and Sanofi. Andrew Chan reports receiving honoraria for presentations and advisory board participation to support hospital research funds from Actelion, Almirall, Bayer, Biogen, Celgene, Merck, Novartis, Roche, Sanofi, and Teva; and research support from Biogen, Sanofi, and UCB; and serving as associate editor for the European Journal of Neurology. Dominique Dive reports institutional honoraria for advisory board participation and travel grants from Bayer, Biogen, Merck Serono, Novartis, Roche, Sanofi, and Teva. Sara Eichau reports compensation as a speaker and/or consultant from Biogen, Merck Serono, Novartis, Roche, Sanofi, and Teva. Daniel Kantor reports fees for non-CME/CE services received directly from commercial interests or their agents at AbbVie, Actelion, Bayer, Biogen, Celgene, Corrona, EMD Serono, Genentech, Mylan, Novartis, and Sanofi; and research grants from Actelion and Sanofi. Ho Jin Kim reports receiving a grant from the National Research Foundation of Korea, and research support from AprilBio and Eisai; consultancy/ speaker fees from Alexion, AprilBio, Biogen, Celltrion, Daewoong, Eisai, GC Pharma, HanAll BioPharma, MDimune, Merck Serono, Novartis, Roche, Sanofi Genzyme, Teva-Handok, UCB, and Viela Bio; is a co-editor for the Multiple Sclerosis Journal, and an associate editor for the Journal of Clinical Neurology. Jan Lycke reports receiving travel support and lecture honoraria from Alexion, Biogen, Novartis, Roche, Sanofi, and Teva; compensation as a scientific advisory board participant from Alexion, Biogen, BristolMyers Squibb, Celgene, Novartis, Roche, Sanofi, and Teva; serving on the editorial board of Acta Neurologica Scandinavica; and unconditional research grants from Biogen, Novartis, and Teva. Richard A. L. Macdonell reports participation on scientific advisory boards for Biogen, Merck, Novartis, Roche, Sanofi, and Teva. Carlo Pozzilli reports consulting and/or speaking fees, research, and travel grants from Actelion, Almirall, Biogen, Merck, Novartis, Roche, Sanofi, and Teva. Thomas Scott reports research and consulting/speaking fees from Biogen, Genentech, and Novartis; consulting/speaking fees from Genzyme; and serving as associate editor for BMC Neurology. Basil Sharrack reports receiving advisory board fees from Biogen, Merck, Novartis, Roche, and Sanofi, and research grants from Biogen and Merck. Heinz Wiendl reports consulting and/or speaking fees and grant/research support from Bayer, Bayer Schering, Biogen, Elan Corporation, Lilly, Lundbeck, Merck Serono, Novartis, Novo Nordisk, Sanofi, and Teva Neuroscience. Luke Chung, Nadia Daizadeh, and Darren P. Baker report personal compensation as employees of Sanofi. Patrick Vermersch reports consulting and/or speaking fees and research support from Biogen, Celgene, Merck Serono, Novartis, Roche, Sanofi, and Teva.

Compliance with Ethics Guidelines. The CARE-MS I and II studies, the CARE-MS extension, and the TOPAZ extension were registered with ClinicalTrials.gov (NCT00530348, NCT00548405, NCT00930553, and NCT02255656, respectively). The studies were conducted in accordance with the ethical principles outlined in the Declaration of Helsinki. The study protocol, informed consent forms, and other study-related documents were reviewed and approved by the local independent ethics committees and institutional review boards. Written informed consent to participate in the studies was obtained from all patients.

Data Availability. Qualified researchers may request access to patient-level data and related study documents including the clinical study report, study protocol with any amendments, blank case report form, statistical analysis plan, and data set specifications. Patientlevel data will be anonymized and study documents will be redacted to protect the privacy of trial participants. Further details on Sanofi's data-sharing criteria, eligible studies, and process for requesting access can be found at https://www.clinicalstudydatarequest.com.

Open Access. This article is licensed under a Creative Commons Attribution-NonCommercial 4.0 International License, which permits any non-commercial use, sharing, adaptation, distribution and reproduction in any medium 
or format, as long as you give appropriate credit to the original author(s) and the source, provide a link to the Creative Commons licence, and indicate if changes were made. The images or other third party material in this article are included in the article's Creative Commons licence, unless indicated otherwise in a credit line to the material. If material is not included in the article's Creative Commons licence and your intended use is not permitted by statutory regulation or exceeds the permitted use, you will need to obtain permission directly from the copyright holder. To view a copy of this licence, visit http://creativecommons.org/licenses/bync/4.0/.

\section{REFERENCES}

1. Conradsson D, Ytterberg C, von Koch L, Johansson $S$. Changes in disability in people with multiple sclerosis: a 10-year prospective study. J Neurol. 2018;265(1):119-26.

2. Sutliff MH. Contribution of impaired mobility to patient burden in multiple sclerosis. Curr Med Res Opin. 2010;26(1):109-19.

3. Chalmer TA, Baggesen LM, Norgaard M, et al. Early versus later treatment start in multiple sclerosis: a register-based cohort study. Eur J Neurol. 2018;25(10):1262-e110.

4. Stankiewicz JM, Weiner HL. An argument for broad use of high efficacy treatments in early multiple sclerosis. Neurol Neuroimmunol Neuroinflamm. 2020;7(1):e636.

5. Harding $\mathrm{K}$, Williams $\mathrm{O}$, Willis $\mathrm{M}$, et al. Clinical outcomes of escalation vs early intensive diseasemodifying therapy in patients with multiple sclerosis. JAMA Neurol. 2019;76(5):536-41.

6. Cohen JA, Coles AJ, Arnold DL, et al. Alemtuzumab versus interferon beta $1 \mathrm{a}$ as first-line treatment for patients with relapsing-remitting multiple sclerosis: a randomised controlled phase 3 trial. Lancet. 2012;380(9856):1819-28.

7. Coles AJ, Twyman CL, Arnold DL, et al. Alemtuzumab for patients with relapsing multiple sclerosis after disease-modifying therapy: a randomised controlled phase 3 trial. Lancet. 2012;380(9856): 1829-39.
8. Giovannoni G, Cohen JA, Coles AJ, et al. Alemtuzumab improves preexisting disability in active relapsing-remitting MS patients. Neurology. 2016;87(19):1985-92.

9. Havrdova E, Arnold DL, Cohen JA, et al. Alemtuzumab CARE-MS I 5-year follow-up: durable efficacy in the absence of continuous MS therapy. Neurology. 2017;89(11):1107-16.

10. Coles AJ, Cohen JA, Fox EJ, et al. Alemtuzumab CARE-MS II 5-year follow-up: efficacy and safety findings. Neurology. 2017;89(11):1117-26.

11. Comi G, Alroughani R, Bass AD, et al. Alemtuzumab maintains efficacy on clinical and MRI disease activity outcomes, including slowing of brain volume loss, over 9 years in RRMS patients: CARE-MS II follow-up (TOPAZ study). Presented at: 35th Congress of the European Committee for Treatment and Research in Multiple Sclerosis (ECTRIMS), Stockholm, Sweden, September 11-13 2019.

12. Montalban X, Arnold DL, Boyko AN, et al. Alemtuzumab maintains efficacy on clinical and MRI disease activity outcomes, including slowing of brain volume loss, over 9 years in RRMS patients: CARE-MS I follow-up (TOPAZ study). Presented at: 35th Congress of the European Committee for Treatment and Research in Multiple Sclerosis (ECTRIMS), Stockholm, Sweden, September 11-13 2019.

13. Ziemssen $\mathrm{T}$, Bass AD, Berkovich R, et al. Efficacy and safety of alemtuzumab through 9 years of follow-up in patients with highly active disease: post hoc analysis of CARE-MS I and II patients in the TOPAZ extension study. CNS Drugs. 2020;34(9):973-88.

14. Bielekova B, Tintore M. Sustained reduction of MS disability: new player in comparing disease-modifying treatments. Neurology. 2016;87(19):1966-7.

15. Johnson KP, Brooks BR, Cohen JA, et al. Copolymer 1 reduces relapse rate and improves disability in relapsing-remitting multiple sclerosis: results of a phase III multicenter, double-blind placebo-controlled trial. The Copolymer 1 Multiple Sclerosis Study Group. Neurology. 1995;45(7):1268-76.

16. Phillips JT, Giovannoni G, Lublin FD, et al. Sustained improvement in Expanded Disability Status Scale as a new efficacy measure of neurological change in multiple sclerosis: treatment effects with natalizumab in patients with relapsing multiple sclerosis. Mult Scler. 2011;17(8):970-9.

17. Hauser SL, Bar-Or A, Comi G, et al. Ocrelizumab versus interferon beta- $1 \mathrm{a}$ in relapsing multiple sclerosis. N Engl J Med. 2017;376(3):221-34.

18. Le Page E, Leray E, Taurin G, et al. Mitoxantrone as induction treatment in aggressive relapsing 
remitting multiple sclerosis: treatment response factors in a 5 year follow-up observational study of 100 consecutive patients. J Neurol Neurosurg Psychiatry. 2008;79(1):52-6.

19. Alsop JC, Bergvall N, Cornelissen C, Vormfelde SV, Medin J, Ziemssen T. Confirmed disability improvement in patients with active multiple sclerosis treated with fingolimod versus brace: a matched comparison of treatments from the PANGAEA and PEARL registry studies. Value Health. 2015;18: A750.

20. Kurtzke JF. Patterns of neurologic involvement in multiple sclerosis. Neurology. 1989;39(9):1235-8.

21. Gandek B, Sinclair SJ, Kosinski M, Ware JE Jr. Psychometric evaluation of the SF-36 health survey in Medicare managed care. Health Care Financ Rev. 2004;25(4):5-25.

22. Ware J Jr, Kosinski M, Bjorner J, Turner-Bowker D, Gandek B, Maruish M. User's manual for the 36v2 ${ }^{\circledR}$ Health Survey. Lincoln: Quality Metric; 2007.

23. Arroyo R, Bury DP, Guo JD, et al. Impact of alemtuzumab on health-related quality of life over 6 years in CARE-MS II trial extension patients with relapsing-remitting multiple sclerosis. Mult Scler. 2020;26(8):955-63.

24. EuroQoL Research Foundation. EQ-5D-3L user guide. 2018. https://euroqol.org/publications/userguides. Accessed Aug 25, 2020.

25. Cella DF, Dineen K, Arnason B, et al. Validation of the functional assessment of multiple sclerosis quality of life instrument. Neurology. 1996;47(1): 129-39.

26. Kalincik T, Cutter G, Spelman T, et al. Defining reliable disability outcomes in multiple sclerosis. Brain. 2015;138(Pt 11):3287-98.

27. Leray E, Yaouanq J, Le Page E, et al. Evidence for a two-stage disability progression in multiple sclerosis. Brain. 2010;133(Pt 7):1900-13.

28. Confavreux C, Vukusic S, Moreau T, Adeleine P. Relapses and progression of disability in multiple sclerosis. N Engl J Med. 2000;343(20):1430-8.

29. Scott TF, Hackett CT, Quigley MR, Schramke CJ. Relapsing multiple sclerosis patients treated with disease modifying therapy exhibit highly variable disease progression: a predictive model. Clin Neurol Neurosurg. 2014;127:86-92.

30. Paz Soldan MM, Novotna M, Abou Zeid N, et al. Relapses and disability accumulation in progressive multiple sclerosis. Neurology. 2015;84(1):81-8.
31. Lizak N, Lugaresi A, Alroughani R, et al. Highly active immunomodulatory therapy ameliorates accumulation of disability in moderately advanced and advanced multiple sclerosis. J Neurol Neurosurg Psychiatry. 2017;88(3):196-203.

32. Goldman MD, Motl RW, Rudick RA. Possible clinical outcome measures for clinical trials in patients with multiple sclerosis. Ther Adv Neurol Disord. 2010;3(4):229-39.

33. Frischer JM, Bramow S, Dal-Bianco A, et al. The relation between inflammation and neurodegeneration in multiple sclerosis brains. Brain. 2009;132(Pt 5):1175-89.

34. Coles AJ, Arnold DL, Bass AD, et al. Efficacy and safety of alemtuzumab over 6 years: final results of the 4-year CARE-MS extension trial. Ther Adv Neurol Disord. 2020. https://doi.org/10.1177/ 1756286420982134.

35. Jones J, Anderson J, Phuah C, et al. Improvement in disability after alemtuzumab treatment of multiple sclerosis is associated with neuroprotective autoimmunity. Brain. 2010;133(8):2232-47.

36. Scott TF, You X, Foulds P. Functional system scores provide a window into disease activity occurring during a multiple sclerosis treatment trial. Neurol Res. 2011;33(5):549-52.

37. Scott $\mathrm{T}$, Wang $\mathrm{P}$, You X, Mann M, Sperling B. Relationship between sustained disability progression and functional system scores in relapsingremitting multiple sclerosis: analysis of placebo data from four randomized clinical trials. Neuroepidemiology. 2015;44(1):16-23.

38. Fox EJ, Wynn D, Coles AJ, Palmer J, Margolin DH, CAMMS Investigators. Alemtuzumab improves neurological functional systems in treatment-naive relapsing-remitting multiple sclerosis patients. J Neurol Sci. 2016;363:188-94.

39. Hauser SL, Bar-Or A, Cohen JA, et al. Ofatumumab versus teriflunomide in multiple sclerosis. N Engl J Med. 2020;383(6):546-57.

40. Signori A, Boffa G, Bovis F, et al. Prevalence of disability improvement as a potential outcome for multiple sclerosis trials. Mult Scler. 2020. https:// doi.org/10.1177/1352458520936236 ahead of print).

(Online

41. Wiendl H, Spelman T, Butzkueven H, et al. Realworld disability improvement in patients with relapsing-remitting multiple sclerosis treated with natalizumab in the Tysabri Observational Program. Mult Scler. 2020. https://doi.org/10.1177/ 1352458520926869. 